\title{
Morphological factors extraction of upper limb for young female
}

\author{
Xin He, Hongshu Jin* \\ Wuhan textile university, Wuhan, Hubei 430073, China
}

\begin{abstract}
This paper is proposed to extract the morphological factors of upper limb shapes related to the personalized sleeve design. The 36 items of lateral upper limb morphology were measured for 50 young female aged 19 to 22 years old by the photo measurement method. Based on the correlation relationships of morphological variables of upper limb, there are 4 morphological factors of upper limb shapes with eigenvalues above 1 were extracted by the principal component analysis, and the cumulative variance reached $83.504 \%$. Among them, the factors of girth and height of upper limb were relevance to the girths of torso and stature respectively, and the factor of upper limb oblique angle is influenced by the upper body axis inclination, and the arm root height is interpreted as an independent factor describing the arm root shape. These morphological factors provided the references in key feature indicator sifting for upper limb shape subdivisions and the critical parameters in personalized sleeve structure designing.
\end{abstract}

\section{Introduction}

The arm full length is the only parameter about upper limb size for sleeve structure designing and grading in GB/T 1335-2008 "Standard sizing systems for garmentWoman". However, the more structural parameters reflecting the upper limb shape features were needed for garment design responding to the demands of personalized costume consummations.

In the theoretical research field of body type classification, the feature variables of body surface angles of torso ${ }^{[1-2]}$, girth differences, girth ratios ${ }^{[3]}$ are mostly used as the typical indicators for classifications of human body shape. For the morphological feature subdivision of human body ${ }^{[4-5]}$, the statistical analysis methods of the feature extraction, clustering, and combination of typical indicators for describing the morphological differences of human body, which provides a direction on this research of upper limb morphological factors extraction.

For the methods of human body data acquisition, the photo measurement method has the advantages of multi types of human contour feature data acquiring, such as low cost, fast and accurately ${ }^{[6-7]}$. Besides, the lateral contour of human body surface performances the main morphological features ${ }^{[8]}$, which is beneficial to acquiring multiple morphological data of upper limbs. Therefore, the research of upper limb shape subdivision using three upper limb angle variables by the method of photo measurement ${ }^{[9]}$ is uncompleted in description of upper limb morphological characteristics.

In view of these, this paper is aiming to extract the morphological factors of upper limbs by the photo measurement method. The morphological factors are accounting for the differences of upper limb shape, and the typical indicators of each morphological factor are the key variables for upper limb shape subdivisions and the critical parameters in personalized sleeve structure designing.

\section{Upper limb morphological data acquisition}

\subsection{Upper limb morphological measurement method}

The morphological data of upper limb are acquired by the photo measurement method. In addition, the body surface measurement method was aided for the size of upper torso control parts acquiring.

2.1.1. Measurement object. The measurement objects are 50 young female college students aged 19-22 wearing fitted underwear and standing upright naturally, with the upper limbs hanging down naturally.

\subsubsection{Photo shooting and measurement} requirements. The photos for measurements are shot from $10 \mathrm{~m}$ away using the camera model of Canon EOS 5D Mark III and the lens EF24-105 mm. And the center of the lens is on the same horizontal line as the waistline. The photo of right half body for upper limb morphological measuring is shown in Figure 1. At the same time, a benchmark of $50 \mathrm{~cm}$ is placed on the back of the subject as the reference for converting and recording the actual measurement. 


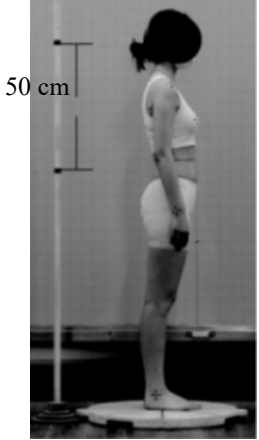

Figure 1. Photo of right half body used for measuring.

\subsection{Measurement items of upper limb shape}

A total of 36 items are measured including 28 items for upper limb and 8 items for upper torso. The photo measurement items are shown in Figure 2.

The measurement items of upper limb include 23 photo measurements such as heights, widths, lengths, and oblique angles; and 5 body surface measurements include arm full length (al), upper-arm girth (uag), elbow girth (e), wrist girth (wr) and armscye girth (ag).

The measurement items of upper torso include 5 photo measurements of bust depth (bd), waist depth (wd), upper back angle $(\angle \mathrm{m})$, lower back angle $(\angle \mathrm{n})$, and upper body axial angle $(\angle \mathrm{o})$; and 3 body surface measurements of stature (h), bust girth (b), and waist girth (w).

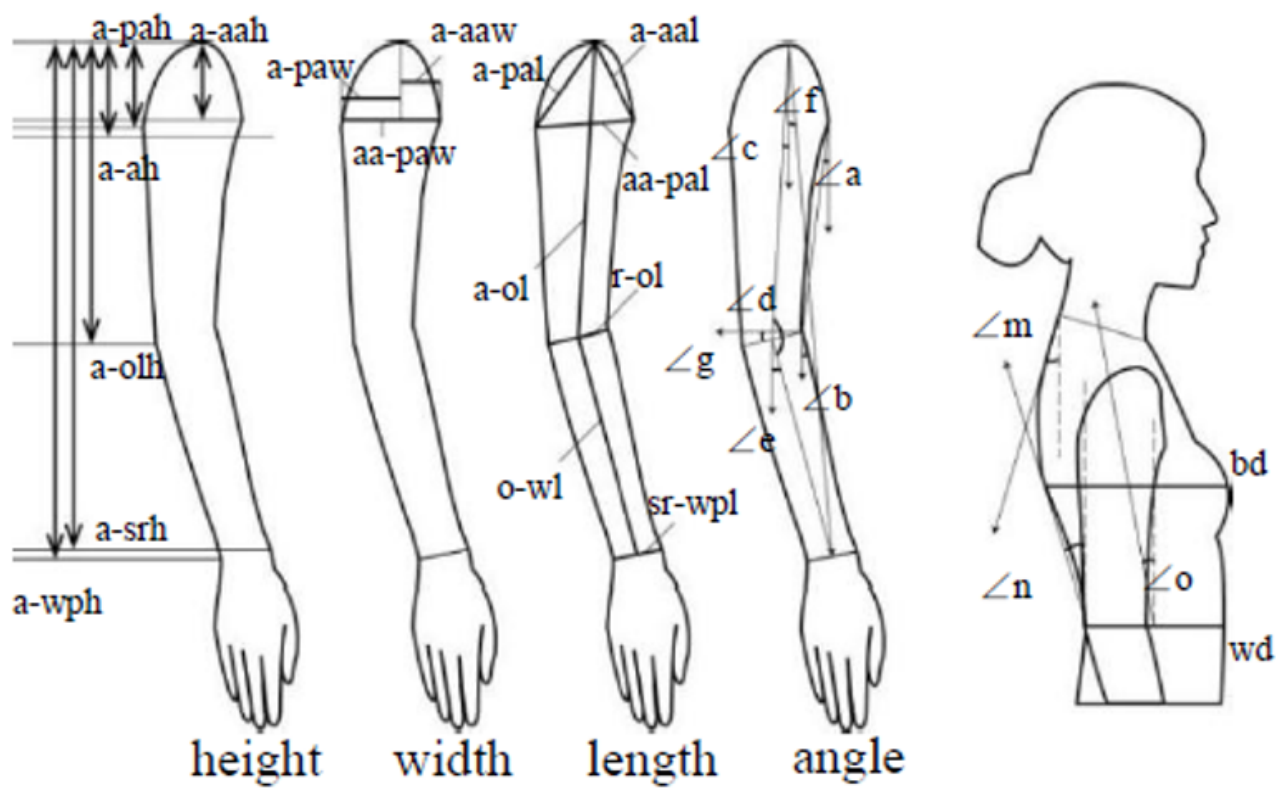

Figure 2. Photo measurement items of upper limb and upper torso.

\section{Basic statistical analysis of upper limb morphological variables}

The results of basic statistics and the single-sample T test of the sizes of control parts were acquired by IBM SPSS Statistics 23 and shown in Table 1. When the test values is the standard measurements of $\mathrm{Y}$ body type in the GB/T 1335.2-2008 "Standard sizing systems for garment-
Woman", the average values of control parts showed significant differences except waist girth (w), which indicated that the stature (h) and arm full length (al) are slightly larger, bust girth (b) is slightly smaller in average than the standard size respectively.

According to the results of Kolmogorov-Smirnov test, the sizes of upper torso control parts and morphological variables of upper limb all follow the normal distribution.

Table 1. Mean test of sample female youth and national standard female data.

\begin{tabular}{ccccc}
\hline Project & $\begin{array}{c}\text { Mean } \\
(\mathrm{cm})\end{array}$ & $\begin{array}{c}\text { Standard deviation } \\
(\mathrm{cm})\end{array}$ & $\begin{array}{c}\text { Test value } \\
(\mathrm{cm})\end{array}$ & $\begin{array}{c}\text { Sig. }(2- \\
\text { tailed })\end{array}$ \\
\hline $\mathrm{h}$ & 161.8 & 4.6 & 160.0 & $* * \mathrm{a}$ \\
$\mathrm{a}$ & 52.4 & 1.9 & 50.5 & $* * \mathrm{a}$ \\
$\mathrm{b}$ & 82.1 & 5.6 & 84.0 & $* \mathrm{~b}$ \\
$\mathrm{a} * * \mathrm{P}<0.01 ;{ }^{\mathrm{b}} \mathrm{P} \mathrm{P}<0.05 ;{ }^{\mathrm{c}}$ No significant & 5.8 & 64.0 & N.S. $^{\mathrm{c}}$ \\
\hline
\end{tabular}




\section{Morphological factors extraction of upper limb shape}

The morphological factors of upper limbs were extracted by principal component analysis based on the correlation coefficients between the morphological variables of upper limbs. Furthermore, the correlated relationships between the variables of upper limbs in each morphological factor and the sizes of upper torso control parts are confirmed.

\subsection{Relationships in upper limb morphological variables}

The heights and lengths of upper limb showed the high significant correlation above $r=0.7$, which indicated the heights and lengths were approximately in described the longitudinal features of upper limbs.

The height of acromion to posterior armpit point (apah) and the height of acromion to armpit horizontal position (a-ah) showed a significant correlation coefficient above $r=0.7$, which described the relationship between the morphological variables of the arm root heights. The width between anterior and posterior armpit points (aa-paw) and armscye girth (ag), upper-arm girth (uag) showed a highly significant correlation above $r=0.8$ respectively, which showed that the arm root widths closely related to the arm root girths. The arm full inclined angle $(\angle \mathrm{f})$ is significantly correlated with the angle of upper arm $(\angle \mathrm{c})$ and angle of lower arm $(\angle \mathrm{e})$ with correlation coefficient above $\mathrm{r}=0.7$, which indicated oblique angle of upper limbs entirely influenced by the full arm oblique angle.

\subsection{Extraction of principal components of upper limb morphological variables}

For extracting the morphological factors of upper limb, the principal component analysis was conducted based on the results of correlation analysis. After removing the variables with weak correlations and showed collinearity between each other, 14 morphological variables of upper limb were sifted and used for analyzing finally.

The results of principal component analysis are shown in Table 2. There are 4 principal components with eigenvalues above 1 were extracted by the maximum variance factor rotation method, and the cumulative variance reached $83.504 \%$. The principle components are explained as follows:

1) In principal component 1 , the variables of the width between anterior and posterior armpit points (aapaw), upper-arm girth (uag), armscye girth (ag), elbow girth (e) showed loading coefficient above 0.8 , which described the features of upper limb girth and width, and it can be summarized as the upper limb girth factor.

2 ) In principal component 2 , the variables with larger loading coefficient included the height of acromion to wrist point (a-wph), arm full length (al), and the length of olecranon to wrist (o-wl), that showed the features of upper limb height, which can be summarized as the upper limb height factor.

3) In principal component 3 , the variables with loading coefficient above 0.7 included arm full inclined angle $(\angle \mathrm{f})$ and angle of upper arm $(\angle \mathrm{c})$, that showed the features of upper limb oblique angle, which can be summarized as the upper limb oblique angle factor.

4) In principal component 4 , the variables with larger loading coefficient included the height of acromion to armpit horizontal position (a-ah) and the height of acromion to posterior armpit point (a-pah), which showed the features of arm root height and summarized as the arm root height factor.

Table 2. Extraction of morphological factors of upper limb after rotation.

\begin{tabular}{|c|c|c|c|c|}
\hline \multirow{2}{*}{ Characteristics of the variables } & \multicolumn{4}{|c|}{ Ingredients } \\
\hline & 1 & 2 & 3 & 4 \\
\hline aa-paw & 0.930 & & & \\
\hline uag & 0.920 & & & \\
\hline ag & 0.892 & & & \\
\hline e & 0.891 & & & \\
\hline a-wph & & 0.962 & & \\
\hline al & & 0.954 & & \\
\hline o-wl & & 0.831 & & \\
\hline a-olh & & 0.746 & & \\
\hline$\angle \mathrm{f}$ & & & 0.988 & \\
\hline$\angle \mathrm{c}$ & & & 0.819 & \\
\hline$\angle \mathrm{e}$ & & & 0.758 & \\
\hline a-ah & & & & 0.842 \\
\hline a-pah & & & & 0.832 \\
\hline $\mathrm{a}-\mathrm{aah}$ & & & & 0.708 \\
\hline
\end{tabular}




\begin{tabular}{ccccc}
\hline Eigenvalue & 3.588 & 3.369 & 2.481 & 2.252 \\
Explain the variance (\%) & 25.632 & 24.065 & 17.722 & 16.082 \\
Cumulative variance (\%) & 25.632 & 46.697 & 67.419 & 83.504 \\
\hline
\end{tabular}

Therefore, the morphological differences of upper limb could be described as 4 factors of upper limb girth, upper limb height, upper limb oblique angle, and arm root height.

\subsection{The relationship between the sizes of upper torso control parts and the morphological variables of upper limb}

For confirming the sizes of upper torso control parts related to morphological variables of upper limbs, the correlation analysis was conducted between the sizes of upper torso control parts and the variables of each upper limb morphological factors.

The bust girth (b) /waist girth (w) showed a significant correlation coefficient with the width between anterior and posterior armpit points (aa-paw) and armscye girth (ag) above $\mathrm{r}=0.7$ respectively. In addition, the bust depth (bd) /waist depth (wd) also showed a significant correlation with the variables of the upper limb girth factor. It could be explained that the girth/width of upper torso control parts were closely related to the size of upper limb.

The stature (h) showed a significant correlation coefficient with the height of acromion to wrist (a-wph) and arm full length (al) above $\mathrm{r}=0.7$ in the upper limb height factor, which explained the upper limb height (length) dimensions influenced by stature obviously.

The upper torso axial angle $(\angle 0)$ significantly correlated with arm full inclined angle $(\angle \mathrm{f})$ in upper limb oblique angle factor above $\mathrm{r}=0.6$, which explained the correlation relationship between the oblique angles of upper limb and upper torso for keeping the balance of upper body.

The arm full length (al) showed a significant correlation coefficient above $\mathrm{r}=0.7$ with the height of acromion to olecranon (a-olh), which indicated that the arm full length is closely related to the heights of upper limb.

To sum up, the factors of girth and height of upper limb were closely related to the sizes of upper torso control parts, which is the references of sleeve size grading. And the factor of upper limb oblique angle is influenced by the upper body axis inclination. In addition, the arm root height factor was the independent factor for arm root shape describing.

\section{Conclusions}

The aim of this paper is to extract the morphological factors of upper limbs by the photo measurement method for young females. The main results summarized as follows:
1) Based on the results of correlation relationships of morphological variables of upper limb, the upper limb shape features interpreted by 4 morphological factors which extracted by principal component analysis.

2) The factors of girth and height of upper limb were relevance to the girths of torso and stature respectively, and the factor of upper limb oblique angle is influenced by and the inclination of upper body axis, and the factor of arm root height is interpreted as an independent factor describing the arm root shape.

These results provided the references in key feature indicator sifting for upper limb shape subdivisions and the critical parameters in personalized sleeve structure designing.

\section{References}

1. Young L, Yun J 2010 Classification of upper lateral body shapes for the apparel industry J. Human Factors and Ergonomics in Manufacturing \& Service Industries. vol 20, no 05. 378-390

2. Wookyung L, Haruki I 2010 Classification of body shape characteristics of women's torsos using angles J. International Journal of Clothing Science and Technology. vol 22, no 04. 297-311

3. He T H, Liu Y N, Zhao X 2018 Body classification and prototype amendment of female college students in Tianjin J. Wool Textile Journal. vol 46, no 07. 5357

4. Xu J Y, Kuang C Y, Liu G L 2008 Research on classification of youth male body based on body surface morphology J. Silk Textile Technology Overseas. no 03. 15-17

5. Sun N L, Song X X 2014 Research on chest classification of young women in Shanghai $\mathrm{J}$. Journal of Silk. vol 51, no 09. 34-39

6. Wang Z J, Dai H 2007 Research on the body parameters influencing the sleeve pattern design of young women garments $\mathrm{J}$. Journal of Xi'an University of Engineering Science and Technology. vol 21, no 01. 48-51

7. Ge Y, Liu G L 2007 Study on photogrammetric measurement of body in apparel industry J. Journal of Textile Research. vol 28, no 10. 78-81

8. Satoru NAKAZAWA 2000 The human body and clothing M. Yuan G L translation, 1st ed (Beijing: China Textile \& Apparel Press) p 176

9. Chen Q, Tuo W, Zhang D 2009 Sleeve structure of men's western-style garment based on arm shape J. Journal of Textile Research. vol 30, no 07. 99-102 